\title{
Breast cancer risk in transgender people receiving hormone treatment: nationwide cohort study in the Netherlands
}

Check for updates

For numbered affiliations see end of the article.

Correspondence to:

M den Heijer, Department of Internal Medicine, Section

Endocrinology, Amsterdam

UMC, VU University Medical

Centre, PO Box 7057, 1007

MB Amsterdam, Netherlands

m.denheijer@vumc.nl

(ORCID 0000-0003-3620-5617)

Additional material is published online only. To view please visit

the journal online.

Cite this as: $B M J$ 2019;365:l1652

http://dx.doi.org/10.1136/bmj.l1652

Accepted: 27 March 2019

\author{
Christel I M de Blok, ${ }^{1,2}$ Chantal M Wiepjes, ${ }^{1,2}$ Nienke M Nota, ${ }^{1,2}$ Klaartje van Engelen, ${ }^{3}$ \\ Muriel A Adank, ${ }^{4}$ Koen M A Dreijerink, ${ }^{1,2}$ Ellis Barbé, ${ }^{5}$ Inge R H M Konings, ${ }^{6}$ Martin den Heijer ${ }^{1,2}$
}

\section{ABSTRACT}

OBJECTIVE

To investigate the incidence and characteristics of breast cancer in transgender people in the Netherlands compared with the general Dutch population.

DESIGN

Retrospective, nationwide cohort study.

\section{SETTING}

Specialised tertiary gender clinic in Amsterdam, the Netherlands.

\section{PARTICIPANTS}

2260 adult trans women (male sex assigned at birth, female gender identity) and 1229 adult trans men (female sex assigned at birth, male gender identity) who received gender affirming hormone treatment.

\section{MAIN OUTCOME MEASURES}

Incidence and characteristics (eg, histology, hormone receptor status) of breast cancer in transgender people.

RESULTS

The total person time in this cohort was 33991 years for trans women and 14883 years for trans men. In the 2260 trans women in the cohort, 15 cases of invasive breast cancer were identified (median duration of hormone treatment 18 years, range 7-37 years). This was 46-fold higher than in cisgender men (standardised incidence ratio $46.7,95 \%$ confidence interval 27.2 to 75.4 ) but lower than in cisgender women $(0.3,0.2$ to 0.4$)$. Most tumours were of ductal origin and oestrogen and progesterone receptor

\section{WHAT IS ALREADY KNOWN ON THIS TOPIC}

Transgender people, defined as an incongruence between sex assigned at birth and experienced gender, can receive gender affirming hormone treatment (sex steroids) to induce desired physical changes

Whether (exogenous) sex steroids influence breast cancer risk and pathogenesis in transgender people is not fully understood

Currently, information about the risk of breast cancer in transgender people is limited

\section{WHAT THIS STUDY ADDS}

An increased risk of breast cancer was observed in trans women (male sex assigned at birth, female gender identity) compared with cisgender men and a lower risk in trans men (female sex assigned at birth, male gender identity) compared with cisgender women

A striking finding was that in trans women the risk of breast cancer increased in a relatively short time

The absolute risk of breast cancer in transgender people remains low, and therefore following breast cancer screening guidelines for cisgender people seems sufficient for transgender people using hormone treatment positive, and $8.3 \%$ were human epidermal growth factor 2 (HER2) positive. In 1229 trans men, four cases of invasive breast cancer were identified (median duration of hormone treatment 15 years, range 2-17 years). This was lower than expected compared with cisgender women (standardised incidence ratio 0.2, 95\% confidence interval 0.1 to 0.5 ).

\section{CONCLUSIONS}

This study showed an increased risk of breast cancer in trans women compared with cisgender men and a lower risk in trans men compared with cisgender women. In trans women, the risk of breast cancer increased during a relatively short duration of hormone treatment and the characteristics of the breast cancer resembled a more female pattern. These results suggest that breast cancer screening guidelines for cisgender people are sufficient for transgender people using hormone treatment.

\section{Introduction}

Breast cancer is the most common malignancy in females, ${ }^{1}$ but it is rare in males. The lifetime risk in the general female population is $12 \%$ and in the general male population is $0.1 \% .^{2}$ Several risk factors have been identified for breast cancer, including advancing age, genetics (eg, BReast CAncer 1/2 mutations (BRCA1/2)), family history, overweight or obesity, breast density, tobacco use, alcohol use, and null parity in females. ${ }^{34}$ The molecular pathogenesis of breast cancer differs between sexes. ${ }^{5}$ For instance, cancers positive for human epidermal growth factor receptor 2 (HER2) are rare in males (1.7\%), whereas $6-12 \%$ of the breast cancers in females express HER2. ${ }^{5-7}$

Transgender people experience an incongruence between the sex assigned to them at birth and their experienced or expressed gender. In the Netherlands, an estimated one in 2800 birth assigned males and one in 5200 birth assigned females identify themselves as transgender. ${ }^{8}$ However, a substantial increase in the number of referrals for psychological counselling, for endocrine or surgical treatment, or a combination of those has been seen over the past 10 years. ${ }^{8}$ Transgender people can receive gender affirming hormones (sex steroids) to reduce psychological distress and to induce desired physical changes, such as for body hair and body composition. ${ }^{9}{ }^{10}$ In trans women (male sex assigned at birth, female gender identity), treatment usually consists of antiandrogens and oestrogens. In trans men (female sex assigned at birth, male gender identity), treatment usually consists of testosterone. Besides gender affirming hormone treatment, transgender people might also want surgical transition, which can consist of breast augmentation 
and orchiectomy or vaginoplasty in trans women and subcutaneous mastectomy and uterus extirpation or oophorectomy, or both, and phalloplasty in trans men. Subcutaneous mastectomy in trans men is often subtotal to obtain an aesthetic masculine thorax.

Whether (exogenous) sex steroids influence breast cancer risk and pathogenesis in transgender people is not fully understood. It is known that sex steroids induce changes in breast tissue. ${ }^{2}{ }^{11}$ During female puberty and in trans women receiving hormone treatment, mammary development includes duct and lobule formation and an increase in deposition of fat in the breasts. ${ }^{2}$ Some structural changes occur in breast tissue under the influence of testosterone, such as an increase in fibrous tissue ${ }^{211}$ and the up regulation of potential oncogenes. ${ }^{12}$ Large prospective studies have shown that hormone replacement therapy increases the risk of breast cancer in cisgender postmenopausal women, ${ }^{13-15}$ in particular in those using both oestrogens and progestogens, which could suggest an increased breast cancer risk in trans women receiving hormone treatment compared with cisgender men.

Currently, information about the risk of breast cancer in transgender people is limited. To date, 22 cases of breast cancer in trans women and 20 cases in trans men have been published. ${ }^{16-19}$ However, reliable estimations of the risk in transgender people are lacking because of the heterogeneity in the population and study cohorts. ${ }^{20}$

To gain more insight into the risk of breast cancer in transgender people receiving hormone treatment and the influence of (exogenous) sex steroids on the development of breast cancer, we investigated the incidence and characteristics of breast cancer in a well documented cohort of transgender people receiving hormone treatment in the Netherlands, compared with the general Dutch population.

\section{Methods}

\section{Study population}

For this retrospective cohort study, we identified all transgender people who visited the gender clinic of the VU University Medical Centre Amsterdam between 1972 and January 2016 for either psychological, endocrine, or surgical treatment. More than 95\% of transgender people in the Netherlands receive healthcare at our centre. ${ }^{8}$ People were excluded from analyses if they never used hormone treatment or the start date was unknown, they were younger than 18 years at the time of the study, or they used alternating oestrogen and testosterone during the follow-up time because of regret about their transition. As data on breast cancer diagnosis were retrieved from the Nationwide Network and Registry of Histopathology and Cytopathology in the Netherlands (PALGA), which covers all pathology diagnoses since $1991,{ }^{21}$ we also excluded those whose last visit to our gender identity clinic was before 1991 .

Most trans women were treated with a combination of antiandrogens and oestrogens. Antiandrogen treatment usually consisted of cyproterone acetate (a progestogenic antiandrogen, 10 to $100 \mathrm{mg}$ daily) or spironolactone (100 to $200 \mathrm{mg}$ daily), and treatment was often stopped after orchiectomy. Oestrogen was prescribed as ethinylestradiol (25 to $100 \mu \mathrm{g}$ daily), conjugated oestrogens (0.625 to $1.25 \mathrm{mg}$ daily), estradiol patches (50 to $150 \mu \mathrm{g} / 24$ hours twice weekly), estradiol implants ( $20 \mathrm{mg}$ every 3 to 6 months), estradiol injections (10 to $100 \mathrm{mg}$ every 2 to 4 weeks), estradiol valerate (2 to $6 \mathrm{mg}$ daily), or estradiol gel (0.75 to $3.0 \mathrm{mg}$ daily). In recent years, mainly estradiol valerate, estradiol patches, or estradiol gel have been used. Trans men were treated with either testosterone gel (20 to $100 \mathrm{mg}$ daily), intramuscular testosterone esters (150 to $250 \mathrm{mg}$ every 2 to 3 weeks), or oral or intramuscular testosterone undecanoate (orally: 40 to $160 \mathrm{mg}$ daily, intramuscularly: $1000 \mathrm{mg}$ every 10 to 14 weeks). Trans men who experienced persistent menstrual blood loss during testosterone treatment were in some cases treated with additional progestogens such as lynestrenol (5 to $10 \mathrm{mg}$ daily). People who started hormone treatment younger than 18 years often received only gonadotrophin releasing hormone agonists before the addition of oestrogen or testosterone treatment.

\section{Data collection}

After inclusion of eligible people, we collected data about age at start of hormone treatment, type of treatment, gender affirming surgery, and medical history. Subsequently, the data were linked to PALGA and Statistics Netherlands (CBS). We retrieved data on year of breast cancer diagnosis and breast cancer histology from PALGA. To adequately calculate the follow-up time, we retrieved data on mortality from Statistics Netherlands.

\section{Statistical analysis}

Data from trans women and trans men were analysed separately. Baseline data are presented as means with standard deviations for normally distributed data, and medians with interquartile ranges, ranges, or both for non-normally distributed data. For those people who had started hormone treatment before treatment at our clinic, we used the first known start date of the treatment to calculate the most accurate duration. To calculate standardised incidence ratios, we determined the number of observed breast cancer cases in this cohort. Person time was calculated as the number of years from the first known start date of hormone treatment to the first terminating event: breast cancer diagnosis, death, or end of study period (31 August 2017). The number of expected cases were calculated using age matched incidence rates for cisgender men and women from the Netherlands Comprehensive Cancer Organisation (IKNL). ${ }^{22}$ We calculated the number of expected cases for the whole study population and for the age categories younger than 30 years, 30 to 50 years, and older than 50 years. Finally, standardised incidence ratios with $95 \%$ confidence intervals were calculated with a mid-P exact test. Mean oestradiol and testosterone concentrations for each participant 
were calculated by averaging the results from the measurements performed during hormone treatment.

Analyses were carried out using STATA statistical software, version 14.1 (Statacorp, College Station, TX) and OpenEpi version 3.01 (www.OpenEpi.com).

\section{Patient and public involvement}

Owing to the design of this study, there was no patient or public involvement. The results of this paper will be shared with the public through our institutions' website and during an open science evening at our centre, which is intended for transgender people, their friends and family, and other interested people.

\section{Results}

Of 6793 transgender people identified, 4432 were birth assigned males and 2361 were birth assigned females. After exclusions, 2260 trans women and 1229 trans men were included in this study (fig 1). The median age at start of hormone treatment in trans women was 31 years (interquartile range 23-41 years) and in trans men was 23 (interquartile range 19-31) years. The median person time in trans women was 13 (interquartile range 5-23, range 0-63) years and in trans men was 8 years (interquartile range 3-20, range 0-47) years. The total person time was 33991 years and 14883 years, respectively. Table 1 shows the baseline characteristics of the study cohort.

In 17 of the 2260 trans women, a total of 18 cases of breast cancer (15 invasive and three non-invasive) were diagnosed after a median 18 (interquartile range 12-27, range 7-37) years of hormone treatment. The median age at diagnosis was 50 (interquartile range 4355) years. The breast cancers were mostly tumours of ductal origin $(67 \%, n=10 / 15)$. The oestrogen receptor was positive in $83 \%(n=10 / 12)$ of the tumours, the progesterone receptor was positive in $67 \%(n=8 / 12)$, and HER2 was positive in $8 \%(n=1 / 12)$. In trans women with breast cancer the median oestradiol level was $236 \mathrm{pmol} / \mathrm{L}$ (range 20-492 pmol/L) and the median testosterone level was $1.3 \mathrm{nmol} / \mathrm{L}$ (range 0.8 $1.3 \mathrm{nmol} / \mathrm{L}$ ), both comparable with the median levels in the whole cohort of trans women (table 1). Table 2 shows the standardised incidence ratios. A higher overall risk of breast cancer was found (standardised

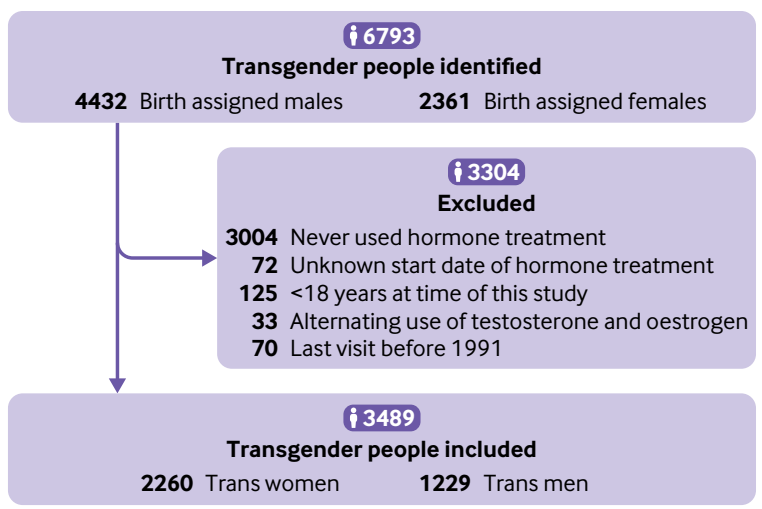

Fig 1 | Study flowchart incidence ratio $46.7,95 \%$ confidence interval 27.2 to 75.4) compared with Dutch cisgender men. A lower overall risk of breast cancer was found compared with Dutch cisgender women (0.3, 0.2 to 0.4$)$.

In four of 1229 trans men, four cases of invasive breast cancer were diagnosed at a median age of 47 (range 35-59) years and after a median 15 (range 2-17) years of hormone treatment, but no cases of noninvasive breast cancer. Three of the four cases of breast cancer were of ductal origin. Two cases were oestrogen and progesterone receptor positive, one was HER2 positive, and one was androgen receptor positive. Three of the four cases of breast cancer were diagnosed several years after subcutaneous mastectomy, the other at the time of mastectomy. The median oestradiol level in trans men with breast cancer was $116 \mathrm{pmol} / \mathrm{L}$ (range 60-191 pmol/L), comparable with the median level in the whole cohort of trans men. The mean testosterone level in trans men with breast cancer was lower than the median level in the whole cohort (13.2 nmol/L (range 12.8-16.0 nmol/L) v 23.3 (15.9-35.2) $\mathrm{nmol} / \mathrm{L}$, respectively). With an overall standardised incidence ratio of 0.2 (95\% confidence interval 0.1 to 0.5 ) in trans men, the risk of breast cancer was lower compared with Dutch cisgender women. Compared with Dutch cisgender men, trans men had a higher overall risk of breast cancer (58.9, 18.7 to 142.2 ). Table 2 shows the standardised incidence ratios.

We were unable to perform analyses on different types of hormone treatment, because treatment was often changed over the follow-up period or there was too little variation in treatment regimen (this was particularly the case for antiandrogen treatment).

\section{Discussion}

This study found an increased risk of breast cancer in trans women in the Netherlands compared with Dutch cisgender men. In both trans women and trans men, the risk of breast cancer was lower than in Dutch cisgender women. This suggests that hormone treatment alters the risk of breast cancer in transgender people compared with initial risk based on their birth assigned sex. The median age at breast cancer diagnosis was 52 years in trans women and 46 years in trans men, both lower than the average age of 61 years in Dutch cisgender women. HER2 receptor status in trans women was higher than expected in male breast cancer.

\section{Comparison with other studies}

The overall incidence of breast cancer for trans women and trans men combined in the current study was 43.0 per 100000 person years, which is higher than the numbers found in two previous studies (20.0 per 100000 person years and 4.5 per 100000 person years). ${ }^{2324}$ Both studies concluded that the risk of breast cancer in transgender people is comparable to the risk in cisgender men. The incidence in the first of the studies, however, was 31.4 per 100000 person years in people who underwent hormone treatment, which 


\begin{tabular}{|c|c|c|c|}
\hline Characteristics & Overall $(n=3489)$ & Trans women $(n=2260)$ & Trans men $(n=1229)$ \\
\hline Age (years) & $47(31-57)$ & $51(38-60)$ & $39(26-51)$ \\
\hline Age at start of hormone treatment (years) & $28(21-38)$ & $31(23-41)$ & $23(19-31)$ \\
\hline$\%$ (No) white & $96.5^{*}(2509)$ & $96.7+(1579)$ & $96.3 \neq(930)$ \\
\hline$\%($ No) ever smokers* & $38.9(1356)$ & $39.1(884)$ & $38.4(472)$ \\
\hline BMI§ & $22.9(20.5-26.2)$ & $22.7(20.4-25.6)$ & $23.2(20.7-27.3)$ \\
\hline \% (No) gonadectomy & - & $68.9(1556)$ & $68.5(842)$ \\
\hline Oestradiol levels (pmol/L) & - & $2179(129-335)$ & $125^{\star \star}(78-176)$ \\
\hline Testosterone levels (nmol/L) & - & $1.3+\dagger(0.8-1.3)$ & $23.3 \neq \ddagger(15.9-35.2)$ \\
\hline Person time (years) & $12(4-22)$ & $13(5-23)$ & $8(3-20)$ \\
\hline Total person time (years) & 48874 & 33991 & 14883 \\
\hline
\end{tabular}

is in line with our results. In the other study, the breast cancer risk could have been underestimated because of technical limitations resulting in overestimation of the total follow-up time and not including people who underwent treatment for breast cancer in other hospitals. We addressed these limitations in our study. None of the reported cases in this study have been previously published.

In accordance with previous studies, we observed a younger age at time of breast cancer diagnosis in transgender people compared with cisgender women. ${ }^{25}$ Moreover, the exposure to hormone treatment before breast cancer diagnosis was relatively short in trans women, at a median of 18 years, suggesting a rapid development of breast tumours in a subset of people. This observation could be explained by genetic susceptibility. Genetics data were available for one trans woman who is a carrier of a BRCA 1 germ line mutation. Another explanation might be the presence of undiagnosed hormone sensitive cancer before treatment, which could become apparent when stimulated by hormones. In trans women, most breast tumours were of the luminal type, suggesting that the tumours are driven in a cell autonomous fashion by the growth stimulatory actions of oestrogens as well as the progestogenic characteristics of the predominantly used antiandrogen cyproterone acetate. As androgens are known to inhibit the progression of luminal breast cancer, antiandrogenic treatment and orchiectomy might have contributed to tumour initiation in the affected people. ${ }^{26}$

\section{Breast cancer screening advise}

Current recommendations suggest that trans women and trans men who have not undergone mastectomy should be biennially screened with mammography from the age of 50 years and if they are using hormone treatment for more than five years. ${ }^{27-29}$ After subcutaneous mastectomy, monitoring of trans men with mammography is not considered feasible owing to the minimal residual breast tissue, and therefore self examination is advised, although there is no evidence for effectiveness. ${ }^{27} 29$ The absolute risk of breast cancer in transgender people is still low in this study, and, more importantly, is not increased compared with cisgender women. We believe therefore that awareness in both doctors and transgender people ${ }^{30}$ is of more importance than the start of screening at a younger age or intensifying available screening, even though the median age at diagnosis in the current study was lower than in cisgender women. Besides, discontinuation of hormone treatment in older transgender people can be considered, which might from then decrease the risk of breast cancer. ${ }^{31}$ Trans women and trans men who have

\begin{tabular}{|c|c|c|c|c|c|}
\hline \multirow[t]{2}{*}{ Variables } & Observed cases & Expected cases & Standardised incidence ratio $(95 \% \mathrm{Cl})$ & Expected cases & Standardised incidence ratio $(95 \% \mathrm{Cl})$ \\
\hline & & \multicolumn{2}{|c|}{ Reference: incidence ratio in cisgender men } & \multicolumn{2}{|c|}{ Reference: incidence ratio in cisgender women } \\
\hline \multicolumn{6}{|c|}{ Trans women $(n=2260)$} \\
\hline Invasive & 15 & 0.32 & $46.7(27.2$ to 75.4$)$ & 59.95 & $0.3(0.2$ to 0.4$)$ \\
\hline \multicolumn{6}{|l|}{ Age (years): } \\
\hline$<30$ & 0 & 0.00 & - & 0.14 & - \\
\hline $30-50$ & 9 & 0.01 & 659.4 (321.6 to 1210.0$)$ & 9.16 & $1.0(0.5$ to 1.8$)$ \\
\hline$>50$ & 6 & 0.31 & $19.5(7.9$ to 40.6$)$ & 50.65 & 0.1 (0.1 to 0.3$)$ \\
\hline Non-invasive & 3 & 0.03 & $96.1(24.5$ to 261.6$)$ & 12.10 & $0.3(0.1$ to 0.7$)$ \\
\hline \multicolumn{6}{|l|}{ Age (years): } \\
\hline$<30$ & 0 & 0.00 & - & 0.01 & - \\
\hline $30-50$ & 1 & 0.00 & 5288.0 (264.6 to 26080.0$)$ & 1.25 & $0.8(0.0$ to 4.0$)$ \\
\hline$>50$ & 2 & 0.03 & $64.5(10.8$ to 213.0$)$ & 10.83 & $0.2(0.0$ to 0.6$)$ \\
\hline \multicolumn{6}{|c|}{ Trans men $(n=1229)$} \\
\hline Invasive & 4 & 0.07 & 58.9 (18.7 to 142.2$)$ & 18.54 & $0.2(0.1$ to 0.5$)$ \\
\hline \multicolumn{6}{|l|}{ Age (years): } \\
\hline$<30$ & 0 & 0.00 & - & 0.14 & - \\
\hline $30-50$ & 2 & 0.01 & $282.3(47.3$ to 932.5$)$ & 4.78 & $0.4(0.1$ to 1.4$)$ \\
\hline$>50$ & 2 & 0.06 & $32.9(5.5$ to 108.8$)$ & 13.62 & $0.2(0.0$ to 0.5$)$ \\
\hline Non-invasive & 0 & 0.01 & - & 3.55 & - \\
\hline
\end{tabular}


not had a mastectomy are advised to undergo the same intensified breast surveillance as their close female relatives if the risk of breast cancer is increased because of a familial predisposition. It is important to remember that transgender people who changed their legal sex might not be automatically invited for population based screenings, including breast cancer screening.

\section{Strengths and limitations of this study}

This study provides novel insights into the risk of breast cancer in transgender people. The reported risk in the current study is higher than estimates from previous studies, possibly related to cohort size and data quality, including the use of a national pathology database. Furthermore, this study included people with a wide range of ages. This study does, however, have some limitations. Owing to the retrospective design of the study, information about hormone use, family history, genetic mutations, benign breast lesions and breast density, tobacco and alcohol use, and body mass index is missing or incomplete. Although these risk factors for breast cancer should not be underestimated, the most important difference between transgender people and cisgender men and women is the use of hormone treatment. It would be interesting to study these risk factors in more detail, to investigate whether certain factors determine the observed increased risk in trans women. The study participants with breast cancer did not necessarily undergo treatment in our centre, and thus detailed data about type and outcome of the treatment are lacking in most of the cases. Although this would be interesting to study in more detail, it was not the purpose of this study. It would be worthwhile for future studies to investigate whether treatment outcomes of breast cancer in trans women are comparable to those of cisgender women.

\section{Conclusions}

This large nationwide cohort study in the Netherlands showed an increased risk of breast cancer in trans women compared with cisgender men. The risk in trans women is still lower than in Dutch cisgender women and resembles a more female type of breast cancer and hormone receptor status. In trans men, a lower risk compared with Dutch cisgender women was observed. Based on this study, we conclude that the absolute overall risk of breast cancer in transgender people remains low and therefore it seems sufficient for transgender people using hormone treatment to follow screening guidelines as for cisgender people. As the risk of breast cancer in trans women increased during a relatively short duration of hormone treatment, it would be worthwhile for future studies to investigate in more detail the cause of breast cancer in transgender people receiving hormone treatment.

\section{AUTHOR AFFILIATIONS}

${ }^{1}$ Centre of Expertise on Gender Dysphoria, Amsterdam UMC, VU University Medical Centre, Amsterdam, Netherlands

${ }^{2}$ Department of Endocrinology, Amsterdam UMC, VU University Medical Centre, Amsterdam, Netherlands
${ }^{3}$ Department of Clinical Genetics, Amsterdam UMC, VU University Medical Centre, Amsterdam, Netherlands

${ }^{4}$ Department of Clinical Genetics, Netherlands Cancer Institute, Amsterdam, Netherlands

${ }^{5}$ Department of Pathology, Amsterdam UMC, VU University Medical Centre, Amsterdam, Netherlands

${ }^{6}$ Department of Oncology, Amsterdam UMC, VU University Medical Centre, Amsterdam, Netherlands

Contributors: $\mathrm{CdB}, \mathrm{CW}, \mathrm{NN}$, and $\mathrm{MdH}$ designed the study. $\mathrm{CdB}, \mathrm{CW}$, $\mathrm{NN}, \mathrm{KvE}, \mathrm{EB}$, and MdH collected the data. CdB, CW, and NN analysed the data. CdB, CW, NN, KvE, MA, KD, EB, IK, and MdH interpreted the data. CdB drafted the manuscript. CdB, CW, NN, KvE, MA, KD, EB, IK, and $\mathrm{MdH}$ revised the manuscript and approved the final version. $\mathrm{CdB}$ and $\mathrm{MdH}$ are the guarantors. This manuscript is an honest, accurate, and transparent account of the study being reported and no important aspects of the study have been omitted. The corresponding author attests that all listed authors meet authorship criteria and that no others meeting the criteria have been omitted.

Funding: None.

Competing interests: All authors have completed the ICMJE uniform disclosure form at www.icmje.org/coi_disclosure.pdf and declare: no support from any organisation for the submitted work; no financial relationships with any organisations that might have an interest in the submitted work in the previous three years; no other relationships or activities that could appear to have influenced the submitted work.

Ethical approval: This study was reviewed by the ethical review board of the VU University Medical Centre Amsterdam. It was determined that the Medical Research Involving Human Subjects Act (WMO) does not apply to this study, and necessity for informed consent was waived. All data were processed anonymously.

Data sharing: Statistics Netherlands prohibit data sharing at an individual level to guarantee the anonymity of the people in its databases.

Transparency: The lead authors ( $\mathrm{CdB}$ and $\mathrm{MdH})$ affirm that the manuscript is an honest, accurate, and transparent account of the study being reported; that no important aspects of the study have been omitted; and that any discrepancies from the study as planned (and, if relevant, registered) have been explained.

This is an Open Access article distributed in accordance with the Creative Commons Attribution Non Commercial (CC BY-NC 4.0) license, which permits others to distribute, remix, adapt, build upon this work non-commercially, and license their derivative works on different terms, provided the original work is properly cited and the use is noncommercial. See: http://creativecommons.org/licenses/by-nc/4.0/.

1 Coleman C. Early Detection and Screening for Breast Cancer. Semin Oncol Nurs 2017;33:141-55. doi:10.1016/j. soncn.2017.02.009

2 Sonnenblick EB, Shah AD, Goldstein Z, Reisman T. Breast Imaging of Transgender Individuals: A Review. Curr Radiol Rep 2018;6:1. doi:10.1007/s40134-018-0260-1

3 Mattingly AE, Kiluk JV, Lee MC. Clinical considerations of risk, incidence, and outcomes of breast cancer in sexual minorities. Cancer Control 2016;23:373-82. doi:10.1177/107327481602300408

4 Johansen Taber KA, Morisy LR, Osbahr AJ3rd, Dickinson BD. Male breast cancer: risk factors, diagnosis, and management (Review)[Review]. Oncol Rep 2010;24:1115-20. doi:10.3892/ or 00000962

5 Fentiman IS. The biology of male breast cancer. Breast 2018;38:1325. doi:10.1016/j.breast.2018.01.001

6 Lakhani SR, Ellis IO, Schnitt SJ, et al. WHO Classification of Tumours of the Breast, 4th edn.

7 van Dooijeweert C, Deckers IAG, Baas IO, van der Wall E, van Diest PJ. Hormone- and HER2-receptor assessment in 33,046 breast cancer patients: a nationwide comparison of positivity rates between pathology laboratories in the Netherlands. Breast Cancer Res Treat 2019; published online 1 Mar. doi:10.1007/s10549-01905180-5

8 Wiepjes CM, Nota NM, de Blok CJM, et al. The Amsterdam Cohort of Gender Dysphoria Study (1972-2015): Trends in Prevalence, Treatment, and Regrets. / Sex Med 2018;15:582-90. doi:10.1016/j. isxm.2018.01.016

9 The World Professional Association of Transgender Health. Standards of care for the health of transsexual, transgender, and gender nonconforming people. Version 7. WPATH, 2012.

10 de Blok CJM, Klaver M, Wiepjes CM, et al. Breast Development in Transwomen After 1 Year of Cross-Sex Hormone Therapy: Results of a Prospective Multicenter Study. J Clin Endocrinol Metab 2018;103:532-8. doi:10.1210/jc.2017-01927 
11 Grynberg M, Fanchin R, Dubost G, et al. Histology of genital tract and breast tissue after long-term testosterone administration in a female-to-male transsexual population. Reprod Biomed Online 2010;20:553-8. doi:10.1016/j.rbmo.2009.12.021

12 Millican-Slater R, Good R, Nash C, et al. Adding value to rare tissue samples donated to biobanks: characterisation of breast tissue and primary cell cultures obtained from a female-to-male transgender patient. Cell Tissue Bank 2015;16:27-34. doi:10.1007/s10561014-9444-y

13 Beral V. Million Women Study Collaborators. Breast cancer and hormone-replacement therapy in the Million Women Study. Lancet 2003:362:419-27 doi:10.1016/S0140-6736(03)14596-5

14 Colditz GA, Hankinson SE, Hunter DJ, et al. The use of estrogens and progestins and the risk of breast cancer in postmenopausal women. N Engl / Med 1995;332:1589-93. doi:10.1056 NEJM199506153322401

15 Rossouw JE, Anderson GL, Prentice RL, et al, Writing Group for the Women's Health Initiative Investigators. Risks and benefits of estrogen plus progestin in healthy postmenopausal women: principal results From the Women's Health Initiative randomized controlled trial. JAMA 2002;288:321-33. doi:10.1001/jama.288.3.321

16 Joint R, Chen ZE, Cameron S. Breast and reproductive cancers in the transgender population: a systematic review. BJOG 2018;125:150512. doi:10.1111/1471-0528.15258

17 Hartley RL, Stone JP, Temple-Oberle C. Breast cancer in transgender patients: A systematic review. Part 1: Male to female. Eur / Surg Oncol 2018;44:1455-62.

18 Barghouthi N, Turner J, Perini J. Breast Cancer Development in a Transgender Male Receiving Testosterone Therapy. Case Rep Endocrinol 2018;2018:3652602.

19 Eismann J, Heng YJ, Fleischmann-Rose K, et al. Interdisciplinary Management of Transgender Individuals at Risk for Breast Cancer: Case Reports and Review of the Literature. Clin Breast Cancer 2019;19:e12-9.

20 Braun H, Nash R, Tangpricha V, Brockman J, Ward K, Goodman M. Cancer in Transgender People: Evidence and Methodological Considerations. Epidemiol Rev 2017;39:93-107. doi:10.1093/ epirev/mxw003
21 Casparie M, Tiebosch ATMG, Burger G, et al. Pathology databanking and biobanking in The Netherlands, a central role for PALGA, the nationwide histopathology and cytopathology data network and archive. Cell Oncol 2007;29:19-24.

22 the Netherlands Comprehensive Cancer Organisation (IKNL). Dutch cancer figures. www.ciifersoverkanker.nl/selecties/dataset_1/ img 5b30c289b4687?language=en [updated 2 Feb 2018. accessed 15 May 2018].

23 Gooren LJ, van Trotsenburg MA, Giltay EJ, van Diest PJ. Breast cancer development in transsexual subjects receiving cross-sex hormone treatment. J Sex Med 2013;10:3129-34. doi:10.1111/ jsm.12319

24 Brown GR, Jones KT. Incidence of breast cancer in a cohort of 5,135 transgender veterans. Breast Cancer Res Treat 2015;149:191-8. doi:10.1007/s10549-014-3213-2

25 Maglione KD, Margolies L, Jaffer S, et al. Breast cancer in male-tofemale transsexuals: use of breast imaging for detection. AJR Am J Roentgenol 2014;203:W735-40. doi:10.2214/AJR.14.12723

26 Chia K, O'Brien M, Brown M, Lim E. Targeting the androgen receptor in breast cancer. Curr Oncol Rep 2015;17:4. doi:10.1007/s11912014-0427-8

27 Deutsch MB, Radix A, Wesp L. Breast Cancer Screening, Management, and a Review of Case Study Literature in Transgender Populations. Semin Reprod Med 2017;35:434-41. doi:10.1055/s-0037-1606103

28 Imborek KL, Graf EM, McCune K. Preventive Health for Transgender Men and Women. Semin Reprod Med 2017;35:426-33. doi:10.1055/s-0037-1604457

29 Phillips J, Fein-Zachary VJ, Mehta TS, Littlehale N, Venkataraman $\mathrm{S}$, Slanetz PJ. Breast imaging in the transgender patient. AJR Am J Roentgenol 2014;202:1149-56. doi:10.2214/AJR.13.10810

30 Dhand A, Dhaliwal G. Examining patient conceptions: a case of metastatic breast cancer in an African American male to female transgender patient. J Gen Intern Med 2010;25:158-61. doi:10.1007/s11606-009-1159-6

31 Sattari M. Breast cancer in male-to-female transgender patients: case for caution. Clin Breast Cancer 2015;15:e67-9. doi:10.1016/j. clbc.2014.08.004 